\title{
Internet of Things (IoT): Towards A Better Healthcare
}

\author{
Chetan G, Devashish Bhat, Sri Chaitanya Dinavahi, Ravi P
}

\begin{abstract}
The significance of Internet of Things (IoT) makes different objects connected and it has also been known as the tech revolution. One of the many applications of IoT is in healthcare to examine the patient's health report, internet of things makes medical technology more efficient and less complicated by giving access to real-time analysis of the patient's health, in which it focuses on acquiring the data regarding the patient's health and eliminate the possible human flaws. In the internet of things, a patient's health statistics get transmitted through various medical equipment through a gateway, where they are stored and monitored. The main challenges in the implementation of the internet of things for healthcare services are checking all patients from different places. Therefore, the internet of things in the healthcare field gives the basic solutions for effective patient monitoring at less cost and also reduces the tensions between patient outcomes and disease management. This paper gives emphases on the different techniques used to monitor the patients while working for the healthcare department.
\end{abstract}

Keywords: IoT, Healthcare, Records, Medical Assistance, Patients, wireless health monitoring, sensors.

\section{INTRODUCTION}

IoT drove social insurance and wellness observing framework empower the remote and consistent checking and examination of the patients, with real significance in incessant sicknesses or conditions like corpulence, diabetes, hypertension, heart failure, asthma, nervousness, elderly consideration and support, and aversion care or wellbeing of a person.The IoT models can perform an extraordinary task in the development of era and the nicely-being or fitness of patients by means of enhancing the character of attention, and fast bringing down the treatment costs and constant journey fees. The IoT-driven social insurance framework utilizes organizing incited biosensors to always gather diverse physiological signs and symptoms to transmit the accrued indicators directly to the cloud diagnostic server (CDS) and the medicinal professionals for in addition examination and medical studies. Besides, the IoT-pushed faraway checking frameworks can noticeably lessen tour and fee looking at applications.

IoT, gathers and transmits the statistics specially from the patients and it additionally makes it achievable to collect,

Revised Version Manuscript Received on August 19, 2019.

Chetan G, Department of Computer Science and Engineering, Vidyavardhaka College of Engineering, Mysuru, Karnataka India(email: : joinchetan1998@gmail.com)

DevashishBhat, Department of Computer Science and Engineering, Vidyavardhaka College of Engineering, Mysuru, Karnataka India.

Sri ChaitanyaDinavahi, Department of Computer Science and Engineering, Vidyavardhaka College of Engineering, Mysuru, Karnataka India.

Ravi P, Department of Computer Science and Engineering, Vidyavardhaka College of Engineering, Mysuru, Karnataka India. record and listen new Data versions quicker and with extra accuracy. As the innovation for amassing, examining and transmitting/sharing facts inside the IoT keeps on developing, with the assistance of sensors, actuators, and extraordinary processing devices. This offers statistics communique blessings. These are frequently connected to networking for statistics transmission.This connected healthcare system emphases on the quick flow of information and enable easy access to diseases/illness such as hypertension, diabetic and cardiac diseases which need constant monitoring. The Internet of Things is highly recognized by various researchers and analysts as one of the most efficient technologies for health monitoring system and it is extremely safe for patients.

The capacity of different tech gadgets to collect facts all by myself disposes of the confinements of human imperfections and it paperwork the statistics clearly and sends it to the restorative professional at whatever point they require it. The mechanization brings down the hazard of human mistake. This kind of arrangement makes use of sensors to collect vital physiological statistics and utilization's methods and allotted computing to have a look at and store the facts and afterward transmits the broke down statistics remotely to the medicinal services divisions for in additional research and survey. It improves the manner in the direction of having a restorative expert stopped by way of at regular intervals of time to test the affected person's nicely-being, as an alternative giving a constant mechanized flow of facts. Thus, it complements the nature of human services with the aid of utilizing consistent attention and by using bringing down the rate of caretaking with the aid of decreasing the requirement for a parental parent to efficiently construct information gathering and exam. Powerful far-flung arrangements related thru the IoT are making it viable for checking the electricity of the sufferers. These preparations would now be capable of being utilized to safely get patient's wellbeing facts from numerous sensors, applying complicated calculations to dissect the facts and afterward transmit it thru remote channels, for healing professionals who can deliver fitting wellbeing audit for the patients.

Furthermore, the IoT is likewise believed to offer a sophisticated connection between gadgets, structures, and offerings that work better than a system to gadget communications in addition to enhances various forms of protocols, domains, and one of a kind programs. More in particular, with most of these characteristics, the IoT will witness a new generation of organizing healthcare services in

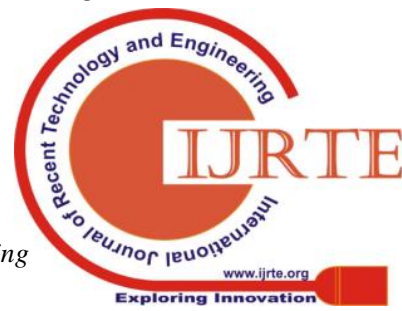


addition to in developing a distinct scientific system.

The remote fitness tracking gadget is being developed with leaps and boundaries with the assist of the Internet of Things (IoT). The goal of a health-tracking machine is to send sufferers fitness-associated records the use of sensors to a far off server or cloud in which the faraway server is effortlessly handy by means of scientific professionals, docs, researchers, hospitals and sufferers, and so on. Anywhere internationally as a simple fundamental of the Internet of Things (IoT).An Expert System and a Decision Support System DSS will further study the sensor outputs using the IoT system and will provide a real-time review to the patient or user immediately. A patient or client will presently be able to access to see or to contrast the present wellbeing estimation and past ones and in the meantime, they can likewise share the wellbeing records with their human services master.

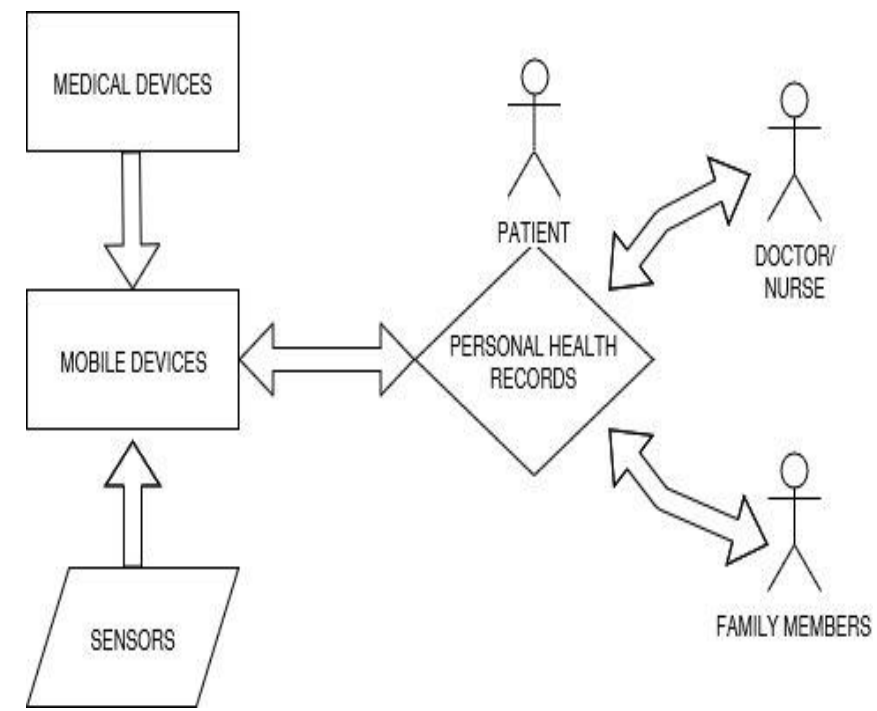

Figure1.1Structure of IOT healthcare

\section{RELATED WORK}

The respiratory rate observing framework gives one of the essential imperative signs which are critical for patients who are conceded in the Intensive Care Unit (ICU). The respiratory rate is determined to utilize the LM35 temperature sensor and is checked through the patient's breath procedure always dependent on the voltage estimation of breathed in and breathed out air or dampness. NRF24101 is additionally used to transmit the information gained from the sensor from home to medicinal consideration focus. At that point, the information is distributed in on web server utilizing Ethernet innovation to know the patient's wellbeing status which is exceptionally valuable for the specialist. And furthermore, the information is shown utilizing Liquid Crystal Display (LCD) screen. When the edge (most extreme limit) is achieved, the caution is consequently produced alongside a message on a page. In this way, the specialist or the restorative expert will come to think about the patient's wellbeing conditions immediately. By utilizing information mining innovation, standard individuals may realize their wellbeing condition without the assistance of a specialist [1].

This paper focuses on analysing the patient's body temperature and heart rate using Arduino equipment's. Health vitals of the patients are studied using IoT which enables the doctor to monitor their patients outside the hospital/clinic and also apart from their consulting hours. Connected health care devices utilize the technology to provide an improved and efficient quality of healthcare, paving the way for better clinical procedures. Benefits of connected medical equivalents reduce clinic visits, and even reduction in bed days of care and long term stays in hospitals. Using IoT, patient conditions can be obtained and recorded for further analysis [2].

In this paper introduces a brand new sign excellent aware IoT enabled ECG telemetry machine for consistent cardiac health monitoring systems. The introduces best conscious ECG monitoring gadget comprises of 3 modules - ECG signal sensing module; automated sign high-quality assessment module; and signal-high-quality conscious ECG evaluation and a transmission module [3].

This paper identified with an android telephone controlled wheel seat alongside an elective utilization of manual joystick. The primary goal is to congratulate and expand the development of individuals who are impaired and the ones who are not ready to move uninhibitedly. Along these lines, a structure of wheelchair which will be an advantage for the therapeutic division and to make it progressively advance in existing innovation, and furnished model with wellbeing observing framework including ongoing estimations of body temperature, pulse, and moistness. This will in the end decline odds of miss-occurring and enables the unfortunate casualty to carry on with a more liberated life [4].

In this paper, they have observed the inpatient and outpatient crucial signs utilizing Healthcare sensor and remote advancements. They have prepared a model and the model is found out by managed learning. Meta information is additionally kept up for finding the wellbeing state of patients. The model restored the status for the given imperative signs, the status will be either sound or not [5].

In this paper, they propose another technique for ECG observing dependent on Cypress Wireless Internet Connectivity for Embedded Devices (WICED) Internet of Things (IoT) stage. ECG information is assembled utilizing a wearable checking hub and is transmitted legitimately to the IoT cloud utilizing Wi-Fi. Web of Things uses open source conventions like CoAP/HTTP, MQTT, TLS/TCP, DTLS/UDP and OMALWM2M for information correspondence and gadget the board. In this work, they planned and actualized an ECG checking framework dependent on bleeding edge cypress WICED IoT innovation. The assembled information was transmitted to the IoT cloud utilizing Wi-Fi, which bolsters high information rates and wide inclusion zones [6].

This paper displays a contextual investigation on a Health-IoT framework where an insightful human services administration is created to screen crucial signs in day by day life. Here, a nonexclusive Health-IoT structure with a Clinical Decision Support System (CDSS) is exhibited. The conventional structure is for the most part centered around the supporting sensors, correspondence media, secure and safe information correspondence, cloud-based capacity, and remote get to of the information. The CDSS is utilized to give 
a customized report on an individual's wellbeing condition dependent on everyday schedule perception on crucial signs [7].

This provides five IoT advances which are basic inside the sending of fruitful IoT-primarily based gadgets and benefits and talks approximately 3 IoT classifications for massive commercial enterprise packages used to improve patron esteem. Also, it seems on the net gift esteem method and the genuine desire methodology typically applied in the legitimization of innovation extends and represents how the real alternative methodology may be connected for IoT task [8].

\section{CASE STUDY ON IOT APPLICATIONS IN HEALTHCARE}

The Internet of Things (IoT) has been having a real effect on every industry, and anyplace this "innovation" cleared with the aid of, you could experience the Midas touch. After the "disclosure" of the Internet, IoT has been making waves that now not a solitary commercial enterprise on the planet can deny or upward push as much as. The folks who do may be for pretty some time abandoned due to the fact the opposition will wind up extra grounded and determined. The uses of IoT within the human services enterprise are numerous. Here are 5 of them:

\section{Real Time Location Services}

Through IoT, specialists can utilize non-stop vicinity administrations and music the gadgets utilized for treating patients. The restorative workforce may additionally now and peer territories which makes them difficult to find out when another therapeutic body of workers is going in advance of the scene. Restorative mechanical meeting and gadgets like wheelchairs, scales, defibrillators, nebulizers, siphons or checking tools can be classified with sensors and found success with IoT. Aside from ongoing area administrations, there are IoT devices that assistance in herbal looking at additionally (checking the fridge temperature, for instance).

Anticipating the Arrival of Patients in PACU

With the mediation of the Internet of Things, clinicians can count on the landing of patients who're improving in the Post-Anaesthesia Care Unit (PACU). They can likewise show the reputation of sufferers progressively.

\section{Hand Hygiene Compliance}

There is hand cleanliness checking frameworks that could understand the extent of neatness in a medicinal offerings labourer. As per the Centre for Disease Control and Prevention within the United States, spherical one affected person out of every 20 get a contamination from the absence of valid hand cleanliness in healing facilities. Various sufferers lose their lives due to medical institution obtained infection. The collaborations within the hand cleanliness looking at frameworks are completed continuously and if a clinician draws close to a patient's bed without washing his arms, the device would possibly start humming. Furthermore, that isn't always all. The data about the medicinal services labourer, his ID, time and location will all be sustained right into a database and this records is probably despatched to the concerned professionals. then keep the devices out of doors of anybody's potential to

\section{Fix Budgets and Improve Patient Journey}

The human services industry desires to be careful for the financial plan and within the interim have refreshed basis to give better affected man or woman encounters. Because of the consistent affiliation among devices that IoT has made feasible, it's miles presently possible for the restorative staff to get too tolerant facts from the cloud so long as they're placed away in there. The goal is to provide first-rate medicinal consideration to sufferers and with the resource of spending a little sum on IT framework medical doctor's centers can provide extraordinary attention to patients at slight charges. IoT plans to offer higher affected person journey with the aid of:

- Room lighting via close to domestic control.

- Communicate to own family and partners thru electronic mail administrations

- Immediate thoughtfulness regarding chronic requirements.

\section{Concentrate at the Research Side of Healthcare}

Protein studies and structure research profits by way of the Internet of Things. Through IoT, scientists can investigate the precision of the equipment, and it rewards them with the aid of shortening their paintings process through a quantitative and reproducible exam of proteins. At the factor whilst a never-ending showcase of devices is associated, the medicinal service's enterprise can give flexible solutions for its patients. Various medicinal offerings programs giving leading edge custom designed arrangements are discharged to them.

\section{DIFFICULTIES OF IOT HEALTHCARE\& RESULTS}

Coming up subsequent are five difficulties of IoT in medicinal services that put it in threat of sadness.

\section{The absence of EHR framework aggregate}

While the data this is collected from IoT devices can comprise a patient's critical symptoms, physical motion or glucose levels whilst at domestic, that statistics do not commonly make a trip to an EHR framework and, quite often, isn't unified or made effectively handy to suppliers. This restricts the statistics an incentive since it isn't always constantly displayed to the provider in a scientific putting.

Interoperability demanding situations preserve IoT facts in various storehouses

Patients are probably going to acquire diverse preparations of information whilst utilizing specific therapeutic devices relying upon each system's motivation and, every now and then, the inquiring for the doctor. A patient with diabetes may every sometimes accumulate glucose tiers and file them again to their vital consideration medical doctor at the same time as likewise conceivably catching statistics identified with their allergies on a specific device, which would possibly move their allergies and sensitivity care dealer. 
IoT facts alone won't be as vital on the off threat that it isn't always in the setting of a complete wellbeing record

Notwithstanding, numerous suppliers bolster the build-up of critical patient information among visits, but, this data is simply profitable inside the event that it has a tendency to be fused and noticed in the setting of a full affected person diagram and route of events. There are as yet several conditions where the data accumulated from wearables and other restorative gadgets stay secured within the IoT service provider storehouse or programs, yet for a medical doctor, that facts won't give any help besides if it's miles obvious in the putting of the patient's complete file.

Information safety causes worries inside the execution of IoT in social insurance

From the time that the records are collected on the system degree to the factor that it's miles transmitted over to its final goal, anchoring that information is primary and is needed beneath HIPAA. In any case, with the absence of fundamental protection pointers and practices, numerous wellbeing IT specialists have concerns about the risks associated with IoT system altering and data breaks.

\section{Steady modifications in equipment and availability innovation}

Patients today require more than one machine to capture the various well-being records their suppliers require. This can require a couple of sensors that, plenty of the time, is utilized nearby a center to which facts are driven that is supposed to technique the facts. These middle factors are not constantly properly with the various sensors which might be on hand and having an absence of primary gadget or remote availability concepts -, as an example, Wi-Fi, Bluetooth or Z-Wave, could make patients have a vast system of their houses, which may be overpowering and costly.

\section{CONCLUSION}

This paper quickly surveys the development of inclines in shrewd medicinal services as an IoT application that has changed the customary restorative framework. Brilliant social insurance frameworks have decreased the intricacy and the confusions related to the utilization of IoT condition. The imperative data in regards to the patient's wellbeing is recorded by the sent IoT objects prompting effective basic leadership. This paper tends to the different manners by which the human services can be given to the needful patient through remote observing, elderly consideration the executives, virtual consultancy and so forth. Likewise, there is a need to give security and protection of the medicinal services information to enhance personal satisfaction of each individual related. This review features the flow difficulties and issues forcing dangers to the scientists. Henceforth an institutionalized system ought to be proposed which will lessen security chance and beat the open issues of compelled situations, cost-viability, versatility and interoperability of human services applications [9].

\section{ACKNOWLEDGEMENTS}

The manuscript is prepared by taking assistance from Accendere Knowledge Management Services Pvt. Ltd, we are thankful to them. We also express our gratitude to our teachers and mentors for guiding us throughout the work.

\section{REFERENCES}

1. A. Raji, P. Kanchana Devi, P. Golda Jeyaseeli, and N. Balaganesh, "Respiratory Monitoring System for Asthma Patients based on IoT" 2016 Online International Conference on Green Engineering and Technologies (IC-GET), pp 1-6, 2016.

2. ChestiAltaffHussian, K. Vuha, M. Rajani, and J. MadhuVineeth, "Smart Health Care Monitoring using Internet of Things and Android" International Journal of Advanced Research in Electronics and Communication Engineering, Vol. 6, No. 3, pp. 101-104, 2017.

3. UditSatija, Barathram.Ramkumar, and $M$. SabarimalaiManikandan, "Real-Time Signal Quality-Aware ECG Telemetry System for IoT-Based Health Care Monitoring", IEEE Internet of Things Journal, Vol. 4, No. 3, 2017.

4. ShubhamSagarNayak, Prateek Gupta, Upasana, and Atul B. Wani, "Wheel Chair with Health Monitoring System Using IoT", International Research Journal of Engineering and Technology, Vol. 4, No. 5, pp. 1063-1067, 2017.

5. T. Saraswathi, and S. Amutha, "IoT Based Wireless Healthcare Monitoring" International Journal of Emerging Trends in Science and Technology, Vol. 3, No. 5, pp. 784-792, 2016.

6. UttamDeshpande, and Milan A. Kulkarni "IoT based Real Time ECG Monitoring System using Cypress WICED”, International Journal of Advanced Research in Electrical, Electronics and Instrumentation Engineering, Vol. 6, No. 2, pp. 710-720, 2017.

7. MobyenUddin Ahmed, "An Intelligent Healthcare Service to Monitor Vital Signs in Daily Life - A Case Study on Health-IoT", International Journal of Engineering Research and Application, Vol. 7, No. 3 (Part-2), pp. 43-55, 2017.

8. In Lee and Kyoochun Lee, "The Internet of Things (IoT): Applications, investments, and challenges for enterprises", Vol. 58, No. 4, pp. 431-440, 2015.

9. AkshayGapchup, AnkitWani,DurveshGapchup, ShashankJadhav, "Health Care Systems Using Internet of Things", International Journal of Innovative Research in Computer and Communication Engineering, Vol. 4, No. 12, pp. 20896-20903, 2016. 